\title{
A quantum information-theoretic proof of the relation between Horn's problem and the Littlewood-Richardson coefficients
}

\author{
Matthias Christandl \\ Centre for Quantum Computation, Department of Applied Mathematics and \\ Theoretical Physics, University of Cambridge, Wilberforce Road, Cambridge \\ CB3 0WA, United Kingdom \\ and \\ Arnold Sommerfeld Center for Theoretical Physics, Faculty of Physics, \\ Ludwig-Maximilians-Universität München, Theresienstr. 37, 80333 München, \\ Germany \\ matthias.christandl@qubit.org
}

\begin{abstract}
Horn's problem asks for the conditions on sets of integers $\mu, \nu$ and $\lambda$ that ensure the existence of Hermitian operators $A, B$ and $A+B$ with spectra $\mu, \nu$ and $\lambda$, respectively. It has been shown that this problem is equivalent to deciding whether $U_{\lambda} \subset U_{\mu} \otimes U_{\nu}$ for irreducible representations of $\operatorname{GL}(d, \mathbb{C})$ with highest weights $\mu, \nu$ and $\lambda$. In this paper we present a quantum information-theoretic proof of the relation between the two problems that is asymptotic in one direction. This result has previously been obtained by Klyachko using geometric invariant theory [1. The work presented in this paper does not, however, touch upon the non-asymptotic equivalence between the two problems, a result that rests on the recently proven saturation conjecture for $\operatorname{GL}(d, \mathbb{C})[2$.
\end{abstract}

\section{Introduction and Results}

Given three spectra $\mu, \nu$ and $\lambda$, are there Hermitian operators $A, B$ with

$$
(\operatorname{Spec} A, \operatorname{Spec} B, \operatorname{Spec} A+B)=(\mu, \nu, \lambda) \quad ?
$$

It is known as Horn's problem to characterise the set of triples $(\mu, \nu, \lambda)$ which have an affirmative answer. Those form a convex polytope whose describing inequalities have been conjectured by Horn in 1962 [3]. In this paper, we will not be concerned with the characterisation of the polytope itself which has by now been achieved [1] 2] but rather with the connection of Horn's problem to the representation theory of $\operatorname{GL}(d, \mathbb{C})$. This connection was first noted by B. V. Lidskii 44 and emerges as a natural twist to Klyachko's work on the inequalities. More precisely, he proves the following two theorems relating the admissible spectral triples to the Littlewood-Richardson coefficients $c_{\mu \nu}^{\lambda} \cdot c_{\mu \nu}^{\lambda}$ is the multiplicity of the irreducible representation $U_{\lambda}$ of $\mathrm{GL}(d, \mathbb{C})$ in the tensor product representation $U_{\mu} \otimes U_{\nu}$ of $\mathrm{GL}(d, \mathbb{C}) . \mu, \nu$ and $\lambda$ denote the highest weights of the respective representations. 
Theorem 1. If $c_{\mu \nu}^{\lambda} \neq 0$, then there exist Hermitian operators $A$ and $B$ such that

$$
(\operatorname{Spec} A, \operatorname{Spec} B, \operatorname{Spec} A+B)=(\mu, \nu, \lambda) .
$$

Theorem 2. For Hermitian operators $A, B$ and $C:=A+B$ with integral spectra $\mu, \nu$ and $\lambda$, there is an $N \in \mathbb{N}$ such that

$$
c_{N \mu, N \nu}^{N \lambda} \neq 0 .
$$

The original proofs of both theorems are based on deep results in geometric invariant theory. The contributions of this paper are elementary quantuminformation-theoretic proofs of Theorem 1 and of the following variant of Theorem 2 ,

Theorem 3. For all Hermitian operators $A, B$ and $C:=A+B$ on $\mathbb{C}^{d}$ with spectra $\mu, \nu$ and $\lambda$, there is a sequence $\left(\mu^{(j)}, \nu^{(j)}, \lambda^{(j)}\right)$, such that

$$
c_{\mu^{(j)} \nu^{(j)}}^{\lambda^{(j)}} \neq 0
$$

and

$$
\begin{aligned}
& \lim _{j \rightarrow \infty} \frac{\mu^{(j)}}{j}=\operatorname{Spec} A \\
& \lim _{j \rightarrow \infty} \frac{\nu^{(j)}}{j}=\operatorname{Spec} B \\
& \lim _{j \rightarrow \infty} \frac{\lambda^{(j)}}{j}=\operatorname{Spec} A+B .
\end{aligned}
$$

Theorems 2 and 3 can be shown to be equivalent with help of the fact that the triples $(\mu, \nu, \lambda)$ with nonvanishing Littlewood-Richardson coefficient form a finitely generated semigroup (see [5] for a similar equivalence in the context of the quantum marginal problem). Here, we choose to prove Theorem 3 since it more naturally fits our quantum information-theoretic approach. The basis of this approach is an estimation theorem for the spectrum of a density operator (Theorem 4) [6], which has recently been used [5] [7] 8] to prove a relation analogous to the one presented in this paper between the Kronecker coefficient of the symmetric group and the spectra of a bipartite density operator and its margins.

In 1999, Knutson and Tao proved the saturation conjecture for $\mathrm{GL}(d, \mathbb{C})$, i.e. they proved that

$$
c_{N \mu, N \nu}^{N \lambda} \neq 0 \text { for some } N \in \mathbb{N} \text { implies } c_{\mu \nu}^{\lambda} \neq 0 .
$$

This result implies that the $N$ in Theorem 2 can be taken to be one and the equivalence of the two problems is strict and not only asymptotic. The proof appeared in [2] and introduces the honeycomb model. A more compact version of this proof based on the hive model was given by [9], and a more accessible discussion can be found in [10].

We proceed with the introduction of the necessary group theory and quantum information theory before turning to the proofs. 


\section{Preliminaries}

\subsection{Spectrum Estimation}

The tensor space $\left(\mathbb{C}^{d}\right)^{\otimes k}$ carries the action of the symmetric group $S_{k}$ which permutes the tensor factors and the diagonal action of $\operatorname{GL}(d, \mathbb{C}): g \mapsto g^{\otimes k}$. Since those actions commute in a maximal way, the tensor space decomposes in a form known as Schur-Weyl duality:

$$
\left(\mathbb{C}^{d}\right)^{\otimes k} \cong \bigoplus_{\lambda} U_{\lambda} \otimes V_{\lambda},
$$

where $U_{\lambda}$ and $V_{\lambda}$ are irreducible representations of $\operatorname{GL}(d, \mathbb{C})$ and $S_{k}$, respectively. The sum extends over all labels $\lambda$ that are partitions of $k$ into $d$ parts, i.e. $\lambda=\left(\lambda_{1}, \ldots, \lambda_{d}\right)$ where the positive integers $\lambda_{i}$ obey $\lambda_{i} \geq \lambda_{i+1}$. As a label of an irreducible representation of $\mathrm{GL}(d, \mathbb{C}), \lambda$ is a dominant weight and as a label of an irreducible representation of $S_{k}$ it is a Young frame.

The following theorem has been discovered by Alicki, Rudnicki and Sadowski in the context of quantum optics [11] and independently by Keyl and Werner for use in quantum information theory [6]. In [7] a short account of Hayashi and Matsumoto's elegant proof [12] of this theorem is given.

Theorem 4. Let $\left(\mathbb{C}^{d}\right)^{\otimes k} \cong \bigoplus_{\lambda} U_{\lambda} \otimes V_{\lambda}$ be the decomposition of tensor space according to Schur-Weyl duality and denote by $P_{\lambda}$ the projection onto $U_{\lambda} \otimes V_{\lambda}$. Then for any density operator $\rho$ with spectrum $r$ we have

$$
\operatorname{Tr} P_{\lambda} \rho^{\otimes k} \leq(k+1)^{d(d-1) / 2} \exp (-k D(\bar{\lambda} \| r))
$$

where $D(\cdot \| \cdot)$ denotes the Kullback-Leibler distance of two probability distributions and $\bar{\lambda}=\left(\bar{\lambda}_{1}, \ldots, \bar{\lambda}_{d}\right)=\left(\frac{\lambda_{1}}{|\lambda|}, \ldots, \frac{\lambda_{d}}{|\lambda|}\right) .|\lambda|=\sum_{i} \lambda_{i}=k$.

This theorem can be interpreted as follows: The joint measurement of $k$ copies of the state $\rho$ by projection onto the spaces $U_{\lambda^{\prime}} \otimes V_{\lambda^{\prime}}$ will - with high probability - result in a measurement outcome $\lambda^{\prime}=\lambda$ satisfying $\frac{\lambda}{k} \approx r$. $\frac{\lambda}{k}$ is therefore an estimate for the spectrum of $\rho$. Indeed the error exponent in eq.(1) is optimal [6].

\subsection{Littlewood-Richardson coefficients}

Given two irreducible representations $U_{\mu}$ and $U_{\nu}$ of $\mathrm{GL}(d, \mathbb{C})$ with highest weights $\mu$ and $\nu$ we decompose the tensor product representation $U_{\mu} \otimes U_{\nu}$ of $\mathrm{GL}(d, \mathbb{C})$ (here, the group is represented simultaneously with $U_{\mu}$ and $U_{\nu}$ ) into irreducible representations of $\mathrm{GL}(d, \mathbb{C})$

$$
U_{\mu} \otimes U_{\nu} \cong \bigoplus_{\lambda} c_{\mu \nu}^{\lambda} U_{\lambda},
$$

where $c_{\mu \nu}^{\lambda}$ denotes the multiplicity of $U_{\lambda}$ and is known as the Littlewood Richardson coefficient. Since $\operatorname{GL}(d, \mathbb{C})$ is the complexification of $U(d)$, the unitary group in $d$ dimensions, we are allowed to - and will later on - regard all 
representations as representations of $U(d)$. The definition of the LittlewoodRichardson coefficient in eq. (2) is indeed the standard one. In the proofs below, however, we will work with a different definition given in terms of the symmetric group:

$$
V_{\lambda} \downarrow_{S_{k} \times S_{n-k}}^{S_{n}} \cong \bigoplus_{\mu, \nu} c_{\mu \nu}^{\lambda} V_{\mu} \otimes V_{\nu}
$$

Here, we restricted the irreducible representation $V_{\lambda}$ of $S_{n}$ to the subgroup $S_{k} \times$ $S_{n-k}$ and decomposed it into products of irreducible representations of $S_{k}$ and $S_{n-k}$. Observing that $S_{n}$ is self-dual, i.e. $V_{\lambda}^{\star} \cong V_{\lambda}$, this definition can be put into the following invariant-theoretic form

$$
c_{\mu \nu}^{\lambda}=\operatorname{dim}\left(V_{\lambda} \otimes V_{\mu} \otimes V_{\nu}\right)^{S_{k} \times S_{n-k}},
$$

where $S_{k} \times S_{n-k}$ acts simultaneously on $V_{\lambda}$ and $V_{\mu} \otimes V_{\nu}$. Clearly, this characterisation only applies to Young frames, i.e. dominant weights with non-negative parts. The extension to the case of arbitrary dominant weights follows from the observation that $c_{\mu \nu}^{\lambda}$ is invariant under the transformation

$$
\begin{aligned}
& \mu \mapsto \mu^{\prime}:=\mu+m\left(1^{d}\right) \\
& \nu \mapsto \nu^{\prime}:=\nu+n\left(1^{d}\right) \\
& \lambda \mapsto \lambda^{\prime}:=\lambda+(m+n)\left(1^{d}\right)
\end{aligned}
$$

for integers $m$ and $n$, i.e. $c_{\mu, \nu}^{\lambda}=c_{\mu^{\prime}, \nu^{\prime}}^{\lambda^{\prime}} .\left(1^{d}\right)$ is short for $(1, \ldots, 1)$ ( $d$ ones).

\section{Proofs}

\subsection{From Hermitian Operators to Density Operators}

It will suffice to prove our results for nonnegative Hermitian operators and Young frames, i.e. dominant weights with nonnegative parts. In order to see this, assume that Theorem 1 holds for Young frames and consider an arbitrary triple of dominant weights $(\mu, \nu, \lambda)$ with $c_{\mu, \nu}^{\lambda} \neq 0$. Choose $m$ and $n$ large enough so that $\left(\mu^{\prime}, \nu^{\prime}, \lambda^{\prime}\right)$ defined above has no negative parts. Since $c_{\mu^{\prime}, \nu^{\prime}}^{\lambda^{\prime}} \neq 0$ there are positive Hermitian operators $A^{\prime}$ and $B^{\prime}$ with

$\left(\operatorname{Spec} A^{\prime}, \operatorname{Spec} B^{\prime}, \operatorname{Spec} A^{\prime}+B^{\prime}\right)=\left(\mu^{\prime}, \nu^{\prime}, \lambda^{\prime}\right)$.

This equation is equivalent to

$(\operatorname{Spec} A, \operatorname{Spec} B, \operatorname{Spec} A+B)=(\mu, \nu, \lambda)$,

where $A:=A^{\prime}-m \mathbb{1}$ and $B:=B^{\prime}-n \mathbb{1}$. The latter is obtained by subtracting $\left(m\left(1^{d}\right), n\left(1^{d}\right),(m+n)\left(1^{d}\right)\right)$ on both sides of the former observing that Spec $\left(A^{\prime}-\right.$ $m \mathbb{1}$ ) = Spec $A^{\prime}-m\left(1^{d}\right)$ (similarly for $B$ ). A similar argument can be carried out for Theorem 3 . 
Since we want to use intuition from quantum information theory, we define $p=\operatorname{Tr} A /(\operatorname{Tr} A+B), \rho^{A}=A / \operatorname{Tr} A$ and $\rho^{B}=B / \operatorname{Tr} B$. The conditions on the spectra of $(A, B, A+B)$ are then equivalent to the conditions on the spectra of $\left(\rho^{A}, \rho^{B}, p \rho^{A}+(1-p) \rho^{B}\right)$, the convex mixture of density operators (i.e. trace one positive Hermitian operators).

We will therefore prove the following two theorems which are equivalent to Theorems 1 and 3 by the above discussion.

Theorem 5. Let $(\mu, \nu, \lambda)$ be a triple of Young frames with $c_{\mu, \nu}^{\lambda} \neq 0$. Then there exist quantum states $\rho^{A}$ and $\rho^{B}$ such that

$$
\begin{aligned}
& \text { Spec } \rho^{A}=\bar{\mu} \\
& \text { Spec } \rho^{B}=\bar{\nu} \\
& \text { Spec } \rho^{C}=\bar{\lambda},
\end{aligned}
$$

where $p=\frac{|\mu|}{|\lambda|}$ and $\rho^{C}=p \rho^{A}+(1-p) \rho^{B}$.

Theorem 6. For all density operators $\rho^{A}, \rho^{B}$ and $\rho^{C}=p \rho^{A}+(1-p) \rho^{B}$ on $\mathbb{C}^{d}$ with spectra $\mu, \nu$ and $\lambda$ and $p \in[0,1]$, there is a sequence $\left(\mu^{(j)}, \nu^{(j)}, \lambda^{(j)}\right)$, such that

$$
c_{\mu^{(j)}, \nu^{(j)}}^{\lambda^{(j)}} \neq 0
$$

and

$$
\begin{aligned}
& \lim _{j \rightarrow \infty} \bar{\mu}^{(j)}=\operatorname{Spec} \rho^{A} \\
& \lim _{j \rightarrow \infty} \bar{\nu}^{(j)}=\operatorname{Spec} \rho^{B} \\
& \lim _{j \rightarrow \infty} \bar{\lambda}^{(j)}=\operatorname{Spec} \rho^{C} .
\end{aligned}
$$

\subsection{Proof of Theorem 5}

We assume without loss of generality that $0<p \leq \frac{1}{2}$. It is well-known that the Littlewood-Richardson coefficients form a semigroup, i.e. $c_{\mu \nu}^{\lambda} \neq 0$ and $c_{\mu^{\prime} \nu^{\prime}}^{\lambda^{\prime}} \neq 0$ implies $c_{\mu+\mu^{\prime}, \nu+\nu^{\prime}}^{\lambda+\lambda^{\prime}} \neq 0[13]$ [14. As a consequence, $c_{\mu \nu}^{\lambda} \neq 0$ implies $c_{N \mu N \nu}^{N \lambda} \neq 0$ for all $N$. For every $N$ we will now construct density operators $\rho_{N}^{A}$ and $\rho_{N}^{B}$ whose $\operatorname{limits} \rho^{A}:=\lim _{N \rightarrow \infty} \rho_{N}^{A}$ and $\rho^{B}:=\lim _{N \rightarrow \infty} \rho_{N}^{B}$ satisfy the claim of the theorem.

Fix a natural number $N$, set $n:=N|\lambda|$ as well as $k:=N|\mu|$ and let $p:=\frac{k}{n}$. Since $c_{\mu \nu}^{\lambda}$ can only be nonzero if $|\mu|+|\nu|=|\lambda|$, we further have $n-k=N|\nu|$. As explained above, the nonnegativity of the parts of $\mu, \nu$ and $\lambda$ allows us to invoke the characterisation of the Littlewood-Richardson coefficient in terms of the symmetric group:

$$
c_{N \mu, N \nu}^{N \lambda}=\operatorname{dim}\left(V_{N \mu} \otimes V_{N \nu} \otimes V_{N \lambda}\right)^{S_{k} \times S_{n-k}},
$$


where $S_{k}$ acts on $V_{N \mu}, S_{n-k}$ on $V_{N \nu}$ and $S_{k} \times S_{n-k} \subset S_{n}$ on $V_{N \lambda}$. Now pick a nonzero $\left|\Psi_{N}\right\rangle \in\left(V_{N \mu} \otimes V_{N \nu} \otimes V_{N \lambda}\right)^{S_{k} \times S_{n-k}}$. Consider

$$
\begin{aligned}
& \mathcal{H}^{(1)} \otimes \cdots \otimes \mathcal{H}^{(k)} \otimes \mathcal{H}^{(k+1)} \otimes \cdots \otimes \mathcal{H}^{(n)} \\
& \otimes \mathcal{K}^{(1)} \otimes \cdots \otimes \mathcal{K}^{(k)} \otimes \mathcal{K}^{(k+1)} \otimes \cdots \otimes \mathcal{K}^{(n)},
\end{aligned}
$$

where $\mathcal{H}^{(i)}$ and $\mathcal{K}^{(j)}$ are isomorphic to $\mathbb{C}^{d}$. Embed the representation $V_{N \mu}$ in $\mathcal{H}^{(1)} \otimes \cdots \otimes \mathcal{H}^{(k)}, V_{N \nu}$ in $\mathcal{H}^{(k+1)} \otimes \cdots \otimes \mathcal{H}^{(n)}$ and $V_{N \lambda}$ in $\mathcal{K}^{(1)} \otimes \cdots \otimes \mathcal{K}^{(n)}$. The symmetric group $S_{n}$ permutes the pairs $\mathcal{H}^{(i)} \otimes \mathcal{K}^{(i)} \cong \mathbb{C}^{d^{2}}$ and its subgroup $S_{k} \times S_{n-k}$ permutes the first $k$ and the last $n-k$ pairs separately.

Any irreducible representation of the group $S_{k} \times S_{n-k}$ is isomorphic to a tensor product of irreducible representations of $S_{k}$ and $S_{n-k} .\left|\Psi_{N}\right\rangle$ is a trivial representation of $S_{k} \times S_{n-k}$ and can therefore only be isomorphic to the tensor product $V_{k} \otimes V_{n-k}$ of the trivial representations $V_{k} \equiv V_{(k, 0, \ldots, 0)}$ of $S_{k}$ and $V_{n-k} \equiv$ $V_{(n-k, 0, \ldots, 0)}$ of $S_{n-k}$. On the first $k$ pairs the $k$-fold tensor product of $g \in \mathrm{U}\left(d^{2}\right)$ commutes with the action of $S_{k}$, and on the remaining pairs it is the $n-k$-fold tensor product of $g \in \mathrm{U}\left(d^{2}\right)$ which commutes with $S_{n-k}$. Schur-Weyl duality decomposes the space in (6) into

$$
\bigoplus_{\tau, \tau^{\prime}} U_{\tau}^{d^{2}} \otimes V_{\tau} \otimes U_{\tau^{\prime}}^{d^{2}} \otimes V_{\tau^{\prime}}
$$

so that

$$
\left|\Psi_{N}\right\rangle \in U_{k}^{d^{2}} \otimes V_{k} \otimes U_{n-k}^{d^{2}} \otimes V_{n-k},
$$

and in terms of projectors onto those spaces

$$
\begin{aligned}
\left|\Psi_{N}\right\rangle\left\langle\Psi_{N}\right| & \leq P_{k} \otimes P_{n-k} \\
& =\left[\operatorname { d i m } U _ { k } ^ { d ^ { 2 } } \int _ { \mathbb { C } P ^ { d ^ { 2 } - 1 } } | \psi \rangle \langle \psi | ^ { \otimes k } d \psi ] \otimes \left[\operatorname{dim} U_{n-k}^{d^{2}} \int_{\mathbb{C} P^{d^{2}-1}}|\phi\rangle\left\langle\left.\phi\right|^{\otimes(n-k)} d \phi\right] .\right.\right.
\end{aligned}
$$

This directly implies

$$
\begin{aligned}
1 & =\operatorname{Tr}\left|\Psi_{N}\right\rangle\left\langle\Psi_{N}\right| P_{k} \otimes P_{n-k} \\
& \leq \operatorname{dim} U_{k}^{d^{2}} \operatorname{dim} U_{n-k}^{d^{2}} \max _{\psi, \phi} \operatorname{Tr}\left|\Psi_{N}\right\rangle\left\langle\Psi_{N}|| \psi\right\rangle\left\langle\left.\psi\right|^{\otimes k} \otimes \mid \phi\right\rangle\left\langle\left.\phi\right|^{\otimes(n-k)}\right.
\end{aligned}
$$

and therefore guarantees the existence of vectors $\left|\phi_{N}\right\rangle$ and $\left|\psi_{N}\right\rangle$ satisfying

$$
\operatorname{Tr}\left[\left|\Psi_{N}\right\rangle\left\langle\Psi_{N}|| \phi_{N}\right\rangle\left\langle\left.\phi_{N}\right|^{\otimes k} \otimes \mid \psi_{N}\right\rangle\left\langle\left.\psi_{N}\right|^{\otimes(n-k)}\right] \geq\left(\operatorname{dim} U_{k}^{d^{2}} \operatorname{dim} U_{n-k}^{d^{2}}\right)^{-1} .\right.
$$

Since $\left|\Psi_{N}\right\rangle\left\langle\Psi_{N}\right| \leq P_{N \mu} \otimes P_{N \nu} \otimes P_{N \lambda}$ we have

$$
\begin{aligned}
& \operatorname{Tr}\left[P_{N \mu} \otimes P_{N \nu} \otimes P_{N \lambda}\right]\left[\left|\phi_{N}\right\rangle\left\langle\left.\phi_{N}\right|^{\otimes p n} \otimes \mid \psi_{N}\right\rangle\left\langle\left.\psi_{N}\right|^{\otimes(1-p) n}\right]\right. \\
& \geq \operatorname{Tr}\left|\Psi_{N}\right\rangle\left\langle\Psi_{N}\right|\left[\left|\phi_{N}\right\rangle\left\langle\left.\phi_{N}\right|^{\otimes p n} \otimes \mid \psi_{N}\right\rangle\left\langle\left.\psi_{N}\right|^{\otimes(1-p) n}\right] .\right.
\end{aligned}
$$

We define

$$
\begin{aligned}
& \rho_{N}^{A}=\operatorname{Tr}_{\mathcal{K}^{(1)}}\left|\phi_{N}\right\rangle\left\langle\phi_{N}\left|=\operatorname{Tr}_{\mathcal{H}^{(1)}}\right| \phi_{N}\right\rangle\left\langle\phi_{N}\right| \\
& \rho_{N}^{B}=\operatorname{Tr}_{\mathcal{K}^{(k+1)}\left|\psi_{N}\right\rangle\left\langle\psi_{N}\left|=\operatorname{Tr}_{\mathcal{H}^{(k+1)}}\right| \psi_{N}\right\rangle\left\langle\psi_{N}\right|}
\end{aligned}
$$


and find, defining $\rho_{N}^{C}=p \rho_{N}^{A}+(1-p) \rho_{N}^{B}$ which satisfies

$$
\operatorname{Tr} P_{N \lambda}\left(\rho_{N}^{C}\right)^{\otimes k} \geq \frac{1}{n+1} \operatorname{Tr} P_{N \lambda}\left(\rho_{N}^{A}\right)^{\otimes p n} \otimes\left(\rho_{N}^{B}\right)^{\otimes(1-p) n},
$$

that

$$
\begin{aligned}
\operatorname{Tr} P_{N \mu}\left(\rho_{N}^{A}\right)^{\otimes p n} & \geq\left(\operatorname{dim} U_{k}^{d^{2}} \operatorname{dim} U_{n-k}^{d^{2}}\right)^{-1} \\
\operatorname{Tr} P_{N \nu}\left(\rho_{N}^{B}\right)^{\otimes(1-p) n} & \geq\left(\operatorname{dim} U_{k}^{d^{2}} \operatorname{dim} U_{n-k}^{d^{2}}\right)^{-1} \\
\operatorname{Tr} P_{N \lambda}\left(\rho_{N}^{C}\right)^{\otimes n} & \geq(n+1)^{-1}\left(\operatorname{dim} U_{k}^{d^{2}} \operatorname{dim} U_{n-k}^{d^{2}}\right)^{-1} .
\end{aligned}
$$

Since $\operatorname{dim} U_{n}^{d^{2}} \leq n^{-d^{2}}$ these are inverse polynomial lower bounds, which, contrasted with the exponential upper bounds from Theorem 4.

$$
\operatorname{Tr} P_{\mu} \rho_{N}^{A \otimes k} \leq(k+1)^{d(d-1) / 2} \exp \left(-k D\left(\bar{\mu} \| r^{A}\right)\right) \leq(k+1)^{d(d-1) / 2} \exp \left(-k \epsilon^{2} / 2\right)
$$

and similarly for $\rho_{N}^{B}$ and $\rho_{N}^{C}$, imply

$$
\begin{array}{r}
\left\|\operatorname{Spec} \rho_{N}^{A}-\bar{\mu}\right\| \leq \epsilon \\
\left\|\operatorname{Spec} \rho_{N}^{B}-\bar{\nu}\right\| \leq \epsilon \\
\left\|\operatorname{Spec} \rho_{N}^{C}-\bar{\lambda}\right\| \leq \epsilon
\end{array}
$$

for $\epsilon=O(d \sqrt{(\log N) / N})$. The proof is now completed, since $N$ was arbitrary and the existence of the limiting operators is guaranteed by the compactness of the set of density operators.

\subsection{Proof of Theorem 6}

We assume without loss of generality that $0<p \leq \frac{1}{2}$. If $p$ is rational, consider a positive integer $n$ such that $k=p n$ (otherwise, approximate $p$ by a sequence of fractions $k / n)$. Define purifications $|\psi\rangle^{A C}$ and $|\phi\rangle^{B C}$ of $\rho^{A}$ and $\rho^{B}$, respectively such that $p \operatorname{Tr}_{A}|\psi\rangle\left\langle\left.\psi\right|^{A C}+(1-p) \operatorname{Tr}_{B} \mid \phi\right\rangle\left\langle\left.\phi\right|^{B C}=\rho^{C}\right.$. Consider the vector

$$
|\tau\rangle=|\psi\rangle^{A_{1} C_{1}} \otimes \cdots \otimes|\psi\rangle^{A_{k} C_{k}} \otimes|\phi\rangle^{B_{1} C_{k+1}} \otimes \cdots \otimes|\phi\rangle^{B_{n-k} C_{n}}
$$

where $|\psi\rangle^{A_{j} C_{j}}=|\psi\rangle^{A C}$ and $|\phi\rangle^{B_{j} C_{k+j}}=|\phi\rangle^{B C}$. This vector is invariant under the action of $S_{k}$ permuting systems $A_{j} C_{j}$ and $S_{n-k}$ permuting the systems $B_{j} C_{k+j}$ and is therefore of the form

$$
|\tau\rangle=\sum_{\mu, \nu, \lambda}\left|\tau_{\mu \nu \lambda}\right\rangle
$$

for vectors $\left|\tau_{\mu \nu \lambda}\right\rangle \in U_{\mu} \otimes U_{\nu} \otimes U_{\lambda} \otimes\left(V_{\mu} \otimes V_{\nu} \otimes V_{\lambda}\right)^{S_{k} \times S_{n-k}}$.

By Theorem 4 , for all $\epsilon>0$ and $\mu$ with $\bar{\mu} \notin \mathcal{B}_{\epsilon}\left(r^{A}\right)=\left\{x:=\left(x_{1}, \ldots, x_{d}\right)\right.$ : $\left.\left\|x-r^{A}\right\|_{1} \leq \epsilon\right\}$ we have

$$
\operatorname{Tr} P_{\mu}\left(\rho^{A}\right)^{\otimes k} \leq(k+1)^{d(d-1) / 2} \exp \left(-k D\left(\bar{\mu} \| r^{A}\right)\right) \leq(k+1)^{d(d-1) / 2} e^{-\frac{k \epsilon^{2}}{2 \ln 2}}
$$


where Pinsker's inequality $D\left(\bar{\mu} \| r^{A}\right) \geq \frac{\left\|\bar{\mu}-r^{A}\right\|_{1}^{2}}{2 \ln 2}$ was used in the last inequality. Similar statements hold for $\rho^{B}$ and $\rho^{C}$. Together with

$$
\begin{aligned}
& \operatorname{Tr} P_{\lambda} \operatorname{Tr}_{A_{1} \cdots A_{k} B_{1} \cdots B_{n-k}}|\tau\rangle\langle\tau|=\operatorname{Tr} P_{\lambda}\left(\operatorname { T r } _ { A } | \psi \rangle \langle \psi | ^ { A C } ) ^ { \otimes k } \otimes \left(\operatorname{Tr}_{B}|\phi\rangle\left\langle\left.\phi\right|^{B C}\right)^{\otimes n-k}\right.\right. \\
& \quad=\operatorname{Tr} P_{\lambda} \frac{1}{n !} \sum_{\pi \in S_{n}} \pi\left(\operatorname { T r } { } _ { A } | \psi \rangle \langle \psi | ^ { A C } ) ^ { \otimes k } \otimes \left(\operatorname{Tr}_{B}|\phi\rangle\left\langle\left.\phi\right|^{B C}\right)^{\otimes n-k} \pi^{-1}\right.\right. \\
& \quad \leq(n+1) \operatorname{Tr} P_{\lambda}\left(\rho^{C}\right)^{\otimes n}
\end{aligned}
$$

we obtain (see [7])

$$
\operatorname{Tr} P_{\mu} \otimes P_{\nu} \otimes P_{\lambda}|\tau\rangle\langle\tau| \leq(n+1)^{d(d-1) / 2}(n+3) e^{-\frac{p n \epsilon^{2}}{2}} .
$$

This estimate can be turned around to give

$$
\sum_{\substack{(\mu, \nu, \lambda):(\bar{\mu}, \bar{\nu}, \bar{\lambda}) \in \\\left(\mathcal{B}_{\epsilon}\left(r^{A}\right), \mathcal{B}_{\epsilon}\left(r^{B}\right), \mathcal{B}_{\epsilon}\left(r^{C}\right)\right)}} \operatorname{Tr} P_{\mu} \otimes P_{\nu} \otimes P_{\lambda}|\tau\rangle\langle\tau| \geq 1-\delta,
$$

for $\delta:=(n+1)^{d(d+8) / 2}(n+3) e^{-\frac{p n \epsilon^{2}}{2 \ln 2}}$, because the number of Young frames with $n$ boxes in $d$ rows is smaller than $(n+1)^{d}$.

For positive RHS of equation (9) the existence of a triple $(\mu, \nu, \lambda)$ with $\| \bar{\mu}-$ $r^{A} \| \leq \epsilon$ (and for $\nu$ and $\lambda$ alike) and $\left|\tau_{\mu \nu \lambda}\right\rangle \neq 0$ is therefore guaranteed. In particular,

$$
c_{\mu \nu}^{\lambda}=\operatorname{dim}\left(V_{\mu} \otimes V_{\nu} \otimes V_{\lambda}\right)^{S_{k} \times S_{n-k}} \neq 0
$$

holds. The proof of the theorem is completed with the choice of an increasing sequence of appropriate integers $n$. The speed of convergence of the resulting sequence of normalised triples to $\left(r^{A}, r^{B}, r^{C}\right)$ can be estimated with $\epsilon=O(d \sqrt{(\log n) / n})$, a value for which the LHS of eq.(9) is bounded away from zero.

\section{Acknowledgment}

The technique used in this paper was developed in collaboration with Graeme Mitchison and Aram Harrow in the context of the quantum marginal problem. I would like to thank both of them for many enlightening discussions. The hospitality of the Accademia di Danimarca in Rome, where part of this work was carried out, is gratefully acknowledged. This work was supported by the European Commission through the FP6-FET Integrated Project SCALA CT-015714, an EPSRC Postdoctoral Fellowship and a Nevile Research Fellowship of Magdalene College Cambridge.

\section{References}

1. Klyachko, A.A.: Stable bundles, representation theory and Hermitian operators. Sel. math. New. ser. 4 (1998) 419-445 
2. Knutson, A., Tao, T.: The honeycomb model of $G L_{n}(C)$ tensor products I: Proof of the saturation conjecture. J. Am. Math. Soc. 12(4) (1999) 1055-1090

3. Horn, A.: Eigenvalues of sums of Hermitian matrices. Pacif. J. Math. 12 (1962) $225-241$

4. Lidskii, B.V.: Spectral polyhedron of the sum of two Hermitian matrices. Func. Anal. Appl. 16 (1982) 139-140

5. Christandl, M., Harrow, A.W., Mitchison, G.: On nonzero Kronecker coefficients and what they tell us about spectra. Comm. Math. Phys. 270(3) (2007) 575-585 quant-ph/0511029

6. Keyl, M., Werner, R.F.: Estimating the spectrum of a density operator. Phys. Rev. A 64(5) (2001) 052311

7. Christandl, M., Mitchison, G.: The spectra of density operators and the Kronecker coefficients of the symmetric group. Comm. Math. Phys. 261(3) (2005) 789-797 quant-ph/0409016

8. Christandl, M.: The Structure of Bipartite Quantum States: Insights from Group Theory and Cryptography. PhD thesis, University of Cambridge (February 2006) quant-ph/0604183

9. Buch, A.: The saturation conjecture (after A. Knutson and T. Tao). Enseign. Math. 46 (2000) 43-60 math.CO/9810180.

10. Knutson, A., Tao, T.: Honeycombs and sums of Hermitian matrices. Notices Amer. Math. Soc. 48 (2001) 175-186 math.RT/0009048

11. Alicki, R., Rudnicki, S., Sadowski, S.: Symmetry properties of product states for the system of $N n$-level atoms. J. Math. Phys. 29(5) (1988) 1158-1162

12. Hayashi, M., Matsumoto, K.: Quantum universal variable-length source coding. Phys. Rev. A 66(2) (2002) 022311

13. Elashvili, A.G.: Invariant algebras. Advances in Soviet Math. 8 (1992) 57-64

14. Zelevinsky, A.: Littlewood-Richardson semigroups. (1997) math.CO/9704228. 